\title{
Editorial: Neoliberale Konterrevolution - Die neue amerikanische Herausforderung auf dem Weltmarkt?
}

Während der Abfassung dieser Zeilen wird in den Nachrichten bekanntgegeben, daß der "Sachverständigenrat zur Begutachtung der gesamtwirtschaftlichen Entwicklung« soeben ein Sondergutachten herausgegeben hat, in dessen Zentrum Vorschläge zum Abbau der Staatsverschuldung stehen. Die von der Bundesregierung für 1981 vorgesehene Neuverschuldung in Höhe von $69 \mathrm{Mrd}$. DM soll nach den Vorstellungen der Sachverständigen um 35 bis $40 \mathrm{Mrd}$. DM reduziert werden. Mit dem Argument, daß die fiskalpolitischen Maßnahmen des Staates die Gefahr steigender Budgetdefizite für die nächsten Jahre nach sich zögen und schon heute eine Vertrauenskrise mit unabsehbaren Folgen für die Währung ausgelöst hätten, wird vom SVR ein rigides Austerity-Programm formuliert, das in seinen Ausmaßen selbst noch die einschneidenden Regelungen des Haushaltsstrukturgesetzes des Krisenjahres 1975 übertrifft. Die vorgeschlagene Reduktion der Sozialausgaben (Kürzungen der Sozialhilfe, der Ausbildungsförderung, des Wohngeldes, der Zuschüsse zur Arbeitslosenversicherung, des Arbeitslosengeldes usw.), die Aufhebung der bislang unbegrenzten Defizithaftung des Bundes gegenüber der Bundesanstalt für Arbeit, die Umschichtung der Steuereinnahmen hin zu einer Erhöhung der Verbrauchssteuern und die Kürzung der staatlichen Personalausgaben bedeuten politisch eine Kampfansage an die in erfolgreichen sozialen Kämpfen erreichten Errungenschaften der sozialen Sicherung und ökonomisch eine Forcierung der »bereinigenden Wirkungen der Krise «. Durch die restriktive und prozyklische Haushaltspolitik soll der zyklische Krisentrend verstärkt werden - und so vor allem die Gewerkschaften für ihre Verweigerung eines Lohnabschlusses in Höhe des Produktivitätszuwachses durch die beschleunigte Freisetzung von Arbeitskräften bestraft werden. Im Zentrum der Neustrukturierung der bürgerlichen Herrschaft steht also eine Veränderung des Verhältnisses von Staat und Gewerkschaften, mit der Tendenz, die etablierten korporativen Strukturen seitens des Staates aufzulösen. Die Desorganisation des Systems der industriellen Beziehungen wird dabei nicht nur zu einer weiteren Belastung des Verhältnisses von Sozialdemokratie und Gewerkschaftsbewegung führen, sondern auch die Notwendigkeit einer Veränderung der gewerkschaftlichen Politik offenlegen.

Die in der Senkung der Sozialleistungen angelegte Reduktion des Kollektivlohns, also des Lohnanteils, der die politisch regulierte und systemnotwendige Reproduktion der Arbeitskraft garantiert, bewirkt nicht nur eine Erhöhung der Mehrwertrate, sondern hat darüberhinaus Bedeutung für den Gesamtreproduktionsprozeß des Kapitals. Da die ansteigenden Staatsdefizite infolge der vermehrten staatlichen Inanspruchnahme der ökonomischen Ressourcen destabilisierende Tendenzen verstärken, ergibt sich systemimmanent der Zwang einer Rückverteilung der Ressourcen vom staatlichen in den privaten Bereich. Infolge des auch die gesamten siebziger Jahre hindurch erfolgenden Anstiegs der Kapitalintensität ist für die Einrichtung eines neuen Arbeitsplatzes eine erhöhte Kapitalsumme erforderlich, die bei den geringen Zuwachsraten der Produktion nur durch eine Ausweitung der Investi- 
tionsquote bereitzustellen ist. Kreislauftheoretisch folgt daraus eine Verringerung der privaten und staatlichen Konsumquote. Das Restriktionsprogramm des SVR zielt also nicht bloß auf eine Behebung der strukturellen Fiskalkrise des Staates, sondern darüberhinaus und vorrangig auf eine Förderung des Akkumulationsprozesses durch die Schaffung neuer Profitanreize. Während ökonomisch die Wiederherstellung der Profitabilität des Kapitals sichergestellt werden soll, kommt dem Abbau der Sozialleistungen die Funktion zu, einen veränderten politischen, sozialen und ideologischen Rahmen für die erneute Verwertung von Kapital zu schaffen.

Das Programm des SVR, das im Verlauf des Sommers in nur wenig modifizierter Form von der Bundesregierung übernommen zu werden droht, reiht sich damit in die Restrukturierungsprozesse ein, wie sie seit der Weltwirtschaftskrise in nahezu allen kapitalistischen Ländern als Vorbedingung für einen neuen und tragfähigen Aufschwung zu beobachten sind. Die Untersuchung dieser Prozesse bilden auch die thematischen Schwerpunkte dieser PROKLA-Ausgabe. In Anknüpfung an Heft 42, in dem versucht wurde, die Krisenmomente zu bestimmen, die gegenwärtig die als Resultat des Zweiten Weltkriegs entstandenen ökonomischen und politischen Strukturen der kapitalistischen »Weltordnung « untergraben und zerstören, sollen in den folgenden Beiträgen die Restukturierungsprozesse und die damit verbundenen theoretischen und strategischen Ansätze einer Analyse unterzogen werden.

Elmar Altvater untersucht in seinem Beitrag die Ursachen der Renaissance der neoliberalen ökonomischen Theorien in den siebziger Jahren und versucht im Anschluß die gemeinsamen Wurzeln des Neoliberalismus und des Konservatismus freizulegen. Die ökonomisch-politische Dimension dieser Strategie wird als eine Individualisierung und Entpolitisierung gesellschaftlicher Probleme und als eine »Entstaatlichung« des »durchstaatlichten Kapitalismus* (K. Renner) interpretiert.

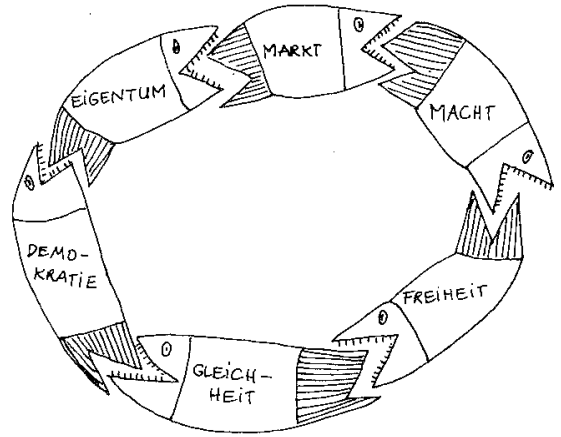

Urs Müller-Plantenberg interpretiert die ökonomische Entwicklung seit Mitte der siebziger Jahre als die Dritte Große Depression des Kapitalismus. Im Vergleich mit den beiden vorhergehenden Großen Depressionen sieht er die mögliche historisch-politische Bedeutung der gegenwärtigen kapitalistischen Entwicklungsphase in der Rückkehr zu einem liberalen Kapitalismus des 19. Jhdts. Am Beispiel Chiles wird gezeigt, welche Bedeutung die staatliche Politik für die Rückführung der ökonomischen und politischen Strukturen in ein solches Kapitalismusmodell hat.

Alex Schubert analysiert in seinem Aufsatz die außenpolitischen Strategien der USA und die möglichen militärischen Bedrohungen nach dem Amtsantritt von Reagan im Kontext der ökonomischen Umstrukturierungen. Und Ferguson / Rogers untersuchen die internen politischen und personellen Verflechtungen zwischen Industrie, Gewerkschaften und politischen Institutionen, die zum Wahlsieg Reagans und der von ihm betriebenen Politik geführt haben.

Chantal Mouffe zeigt die Ursachen für die Massenwirksamkeit der Neoliberalen, Neokonservativen und Neuen Rechten in England, USA und Frankreich, die antistaatliche und 
- bürokratische Ressentiments und populäre Gegenbewegungen gegen die Zerstörung der Familie, Abtreibung, Homosexualität, „Gegenkultur« und »Kollektivismus« geschickt für sich zu nutzen wissen.

Die Verschiebungen auf dem Weltmarkt und die Umorganisation der nationalen Reproduktionsprozesse sind das Thema von Marcel Bübler. Über eine systematische Rezeption neuerer französischer Internationalisierungstheorien wird der bundesrepublikanischen marxistischen Weltmarktdiskussion eine veränderte Analyseperspektive vorgeschlagen. Die Analyse der Bedingungen der Kapitalverwertung zur Erklärung der Weltmarktverschiebungen soll in der Untersuchung der historischen Veränderungen der Reproduktion dominanter Gesellschaftsformationen aufgehoben werden.

Thomas Hurtienne nimmt in seinem Beitrag die Schoeller-Müller-Plantenberg-Debatte über die Überwindung von Abhängigkeit und Unterentwicklung (Heft 42) aus einer anderen Perspektive wieder auf. Durch eine Auseinandersetzung mit den Arbeiten von Dieter Senghaas versucht er der Dependenztheorie bzw. der Theorie des peripheren Kapitalismus ihre analytisch-historische Erklärungskraft zu bestreiten und deren politisch-strategischen Schlußfolgerungen in Frage zu stellen.

Da aus technischen Gründen der Name der Übersetzer des Balibar-Textes aus Heft 43 entfallen ist, möchten wir dies hier nachholen: Wir danken Peter Schöttler und F.O. Wolf für ihre Übersetzungsarbeit.

Die Redaktion

Heft 45 der PROKLA erscheint im Dezember 1981 mit dem Titel »Staat, kein Staat, alternativer Staat? « mit Beiträgen von B. Blanke, H. Gerstenberger, J. Hoffmann, U. Jürgens, P. Schümann, Th. Blanke, R. Hickel u.a.

Heft 46 wird Beiträge zur Entwicklung der Gewerkschaften in der Bundesrepublik enthalten und erscheint in März 1982. 